\title{
Study on the behavioral changes of a post-stroke depression rat model
}

\author{
CHUNFANG WU ${ }^{1}$, JUNHE ZHANG $^{2}$ and YONG CHEN ${ }^{1}$ \\ ${ }^{1}$ Department of Neurology, Henan University Huaihe Hospital, Kaifeng, Henan 475000; \\ ${ }^{2}$ Department of Biochemistry and Molecular Biology, Xinxiang Medical University, Xinxiang, Henan 453003, P.R. China
}

Received July 24, 2014; Accepted April 1, 2015

DOI: $10.3892 /$ etm.2015.2450

\begin{abstract}
The aim of this study was to observe the changes in the levels of learning and memory in a post-stroke depression (PSD) rat model. Forty-eight Wistar rats were randomly divided into three groups: Group A (normal group), group B (stroke group) and group C (PSD group). Each group comprised 16 rats. Three and 7 days after successful modeling, the open-field, sucrose solution consumption and forced swimming tests were performed, so as to evaluate behavioral changes in the three groups of rats. The sucrose solution consumption of the rats in group B was lower than that in group $\mathrm{A}$ in the tests at the two time-points, but only the difference after 7 days was statistically significant $(\mathrm{P}<0.05)$. The sucrose solution consumption of the rats in group $C$ was lower than the consumption of those in groups $\mathrm{A}$ and $\mathrm{B}$, and the differences were statistically significant $(\mathrm{P}<0.05)$. The open-field test results showed that the horizontal and vertical activity scores of the rats in group B were significantly lower than those of the rats in group $\mathrm{A}(\mathrm{P}<0.05)$. Furthermore, the two test levels and the vertical activity scores of the rats in group $\mathrm{C}$ were significantly lower than those of the rats in groups $\mathrm{A}$ and $\mathrm{B}(\mathrm{P}<0.05)$. The immobility time of the rats in the forced swimming test was higher in group B than that in group A at the two time-points, but the difference was only statistically significant after 7 days $(\mathrm{P}<0.05)$. The immobility time of the rats in group $\mathrm{C}$ was significantly higher than that in groups $\mathrm{A}$ and $\mathrm{B}(\mathrm{P}<0.01)$. In conclusion, rats with PSD exhibited a significantly reduced memory capacity and altered behavior. The changes in the PSD rats were more severe than those in the rats in the stroke group.
\end{abstract}

Correspondence to: Dr Yong Chen, Department of Neurology, Henan University Huaihe Hospital, 1 Baogonghu North Road, Kaifeng, Henan 475000, P.R. China

E-mail: yongchencn@163.com

Key words: stroke, post-stroke depression, rat model

\section{Introduction}

Stroke, also known as cerebrovascular accident, is a type of acute cerebral blood circulation disorder with a sudden onset that is caused by cerebral artery stenosis, occlusion or rupture, which may result from a variety of predisposing factors. The clinical manifestations of stroke are indicative of temporary or permanent brain dysfunction (1). Stroke is a common disease among the elderly. According to epidemiological investigation in China, the incidence rate of stroke in middle-aged adults is $200 / 1,000,000$ per year, and the annual mortality rate is $800,000-1,000,000$. There are $1,500,000$ new cases of stroke per year, and $\sim 75 \%$ of the survivors are left disabled. In addition, the recurrence rate in 5 years can reach $40 \%$ (2). In most cases, patients who have been left with a disability following a stroke will also experience further stroke-induced complications; therefore, considerable focus has been placed on researching the recurrence of post-stroke complications (3).

A number of studies have shown that depression is one of the most common post-stroke complications $(4,5)$. The occurrence of depression significantly hinders the rehabilitation of limb function in stroke patients; therefore, over the past two decades, post-stroke depression (PSD) has become an area of significant interest $(3,6)$. Mimicking the pathological and physiological processes of PSD in vivo, by simulating animal models of PSD, is important to enable the thorough elucidation of the occurrence, development and characteristics of PSD and to explore new therapeutic methods for the disease. Several studies have established an animal model of $\operatorname{PSD}(7,8)$, but only a few studies have focused on the behavioral changes in a PSD rat model (9). The aim of the present study, therefore, was to examine and evaluate these changes in a PSD rat model.

\section{Materials and methods}

Experimental animals and grouping. Forty-eight adult Wistar rats (male and female), weighing $200 \pm 20 \mathrm{~g}$, were purchased from the Experimental Animal Center Of Peking University Medical School (Beijing, China). The rats were randomly divided into three groups of 16: Group A (normal group), group B (stroke group) and group C (PSD group). The rats in group A were not treated, the rats in group B were subjected to middle cerebral artery occlusion (MCAO) to generate a stroke 
model and the rats in group $\mathrm{C}$ were used to establish a PSD model following right MCAO. This study was carried out in strict accordance with the guidelines stated in the Guide for the Care and Use of Laboratory Animals of the National Institutes of Health. The animal use protocol was reviewed and approved by the Institutional Animal Care and Use Committee of Henan University Huaihe Hospital (Kaifeng, China).

Establishment of the rat models in groups $B$ and $C$. Right $\mathrm{MCAO}$ was performed in the 32 rats from groups $\mathrm{B}$ and $\mathrm{C}$. All surgeries were performed by the same individual. Wistar rats underwent preoperative fasting for $12 \mathrm{~h}$. Following intraperitoneal anesthesia with amobarbital sodium (Sinopharm Chemical Reagent Co., Shanghai, China), the rats were fixed in a supine position on the operating board and the shaved skin was disinfected. A 2-cm oblique skin incision was made on the midpoint of the ligature between the right lateral canthus and posterior auricular sulcus to expose the temporal arch, and a bone window with a diameter of $0.5 \mathrm{~cm}$ was then opened in front of the temporal arch root to expose the right MCA. Following the exposure of the MCA, the dura mater was opened and the end of the main trunk of the MCA was subjected to bipolar electrocoagulation (Hoboat, Nanjing, China). Soon afterwards the bone window was filled with sterile gelfoam (Hoboat) and the incision was sutured layer by layer. The intraoperative body temperature was maintained at $\sim 37^{\circ} \mathrm{C}$. A postoperative intraperitoneal injection of 40,000 units of penicillin sodium (Shijiazhuang Pharma Group ZhongNuo Pharmaceutical Co. Ltd., Shijiazhuang, China) was performed to prevent infection. The postoperative general state, limb motor function and blood pressure of the rats were closely observed (10) (Fig. 1).

The criteria for a successful MCAO were as follows: i) The reaction to pain was dulled or had disappeared in the affected limbs; ii) the rat was unable to extend its upper limbs when hanging upside down by the tail; iii) the rat slanted toward the affected side when crawling. Motor function was examined weekly following the MCAO surgery, and neurological function was measured using the Bederson score, as follows: i) Score 3, the rat could not crawl forward and exhibited a unidirectional circling motion; ii) score 2 , the rat could move forward but was easily overturned when subjected to a lateral external force on the affected side, and the affected limbs were flexed when the tail was lifted; iii) score 1, the affected forelimbs were flexed when the tail was lifted; iv) score 0 , normal activity (11).

To prepare the rat model of PSD the 16 rats in group C were subjected to MCAO surgery and then fed in isolation and exposed to chronic, unpredictable, mild stress for 2 weeks, as described previously (11).

Behavioral assessment of the rats. Three and 7 days after successful modeling, the forced swimming, open-field and sucrose solution consumption tests were performed on the three groups of rats, and the results were recorded.

For the forced swimming test, water (depth, $18 \mathrm{~cm}$; temperature, $25^{\circ} \mathrm{C}$ ) was injected into a cylindrical container (height, $140 \mathrm{~cm}$; diameter, $17.4 \mathrm{~cm}$ ). Each rat was placed by itself into the container for $5 \mathrm{~min}$. The total length of time that each rat maintained an immobile state in the water was recorded. For the open-field test, an open cylindrical box (height, $40 \mathrm{~cm}$; diameter, $80 \mathrm{~cm}$ ) with a black peripheral wall was used. The bottom surface was composed of 25 blocks of equal area. The rats were placed into the box and their horizontal and vertical activity was assessed. The number of blocks crossed by the rat was counted to give a score of horizontal activity, while the score of vertical activity was determined by counting the number of times the rat reared up on its hind legs. Each rat was assessed once, in isolation, for $5 \mathrm{~min}$. In the sucrose solution consumption experiment the volume of $1 \%$ sucrose solution drunk by each rat in $24 \mathrm{~h}$ was calculated $(12,13)$.

The sucrose solution consumption test was used to evaluate the reward behavior, the open-field test was used to evaluate the vertical and horizontal activity scores and the state of immobility, measured by the forced swimming test, reflected the degree of despair in the rats. All 48 rats of the three groups were tested and no rat died during the tests.

Statistical analysis. Quantitative analysis of all data was performed using SPSS 14.0 software (SPSS Inc., Chicago, IL, USA). The $\chi^{2}$ test was performed to test the significance of heterogeneity in the present study. Correlation analysis for the results of the three tests was carried out. $\mathrm{P}<0.05$ was considered to indicate a statistically significant difference.

\section{Results}

Comparison of the test results of the three groups of rats on day 3 . The sucrose solution consumption of the rats in group B was lower than that in group A, but the difference was not statistically significant $(\mathrm{P}>0.05)$, whereas the sucrose solution consumption of the rats in group $\mathrm{C}$ was significantly lower than that in groups $\mathrm{A}$ and $\mathrm{B}(\mathrm{P}<0.05)$. In the open-field test, the scores of the rats' horizontal and vertical activity were significantly lower in group B than those in group A $(\mathrm{P}<0.05)$; the same scores of the rats in group $\mathrm{C}$ were significantly lower than those of the rats in groups $A$ and $B(P<0.05)$. The results of the forced swimming test showed that the state of immobility of the rats in group B lasted longer than that of the rats in group A, but the difference was not statistically significant $(\mathrm{P}>0.05)$; the state of immobility of the rats in group $\mathrm{C}$ also lasted longer than that of the rats in groups $\mathrm{A}$ and $\mathrm{B}$, but with a statistically significant difference $(\mathrm{P}<0.01)$ (Table I).

Comparison of the test results of the three groups of rats on day 7. The sucrose solution consumption of the rats in group B was significantly lower than that of the rats in group $\mathrm{A}(\mathrm{P}<0.05)$, and the sucrose solution consumption of the rats in group $\mathrm{C}$ was significantly lower than that of the rats in groups $\mathrm{A}$ and $\mathrm{B}(\mathrm{P}<0.05)$. In the open-field test, the scores of the horizontal and vertical activity of the rats in group B were significantly lower than those of the rats in group $\mathrm{A}(\mathrm{P}<0.05)$, and the same scores of the rats in group $\mathrm{C}$ were significantly lower than those of the rats in groups $\mathrm{A}$ and $\mathrm{B},(\mathrm{P}<0.05)$. The results of the forced swimming test showed that the state of immobility of the rats in group B lasted longer than that of the rats in group $\mathrm{A}$, but the difference was not statistically significant $(\mathrm{P}>0.05)$. By contrast, the state of immobility of the rats in group $\mathrm{C}$ lasted longer 

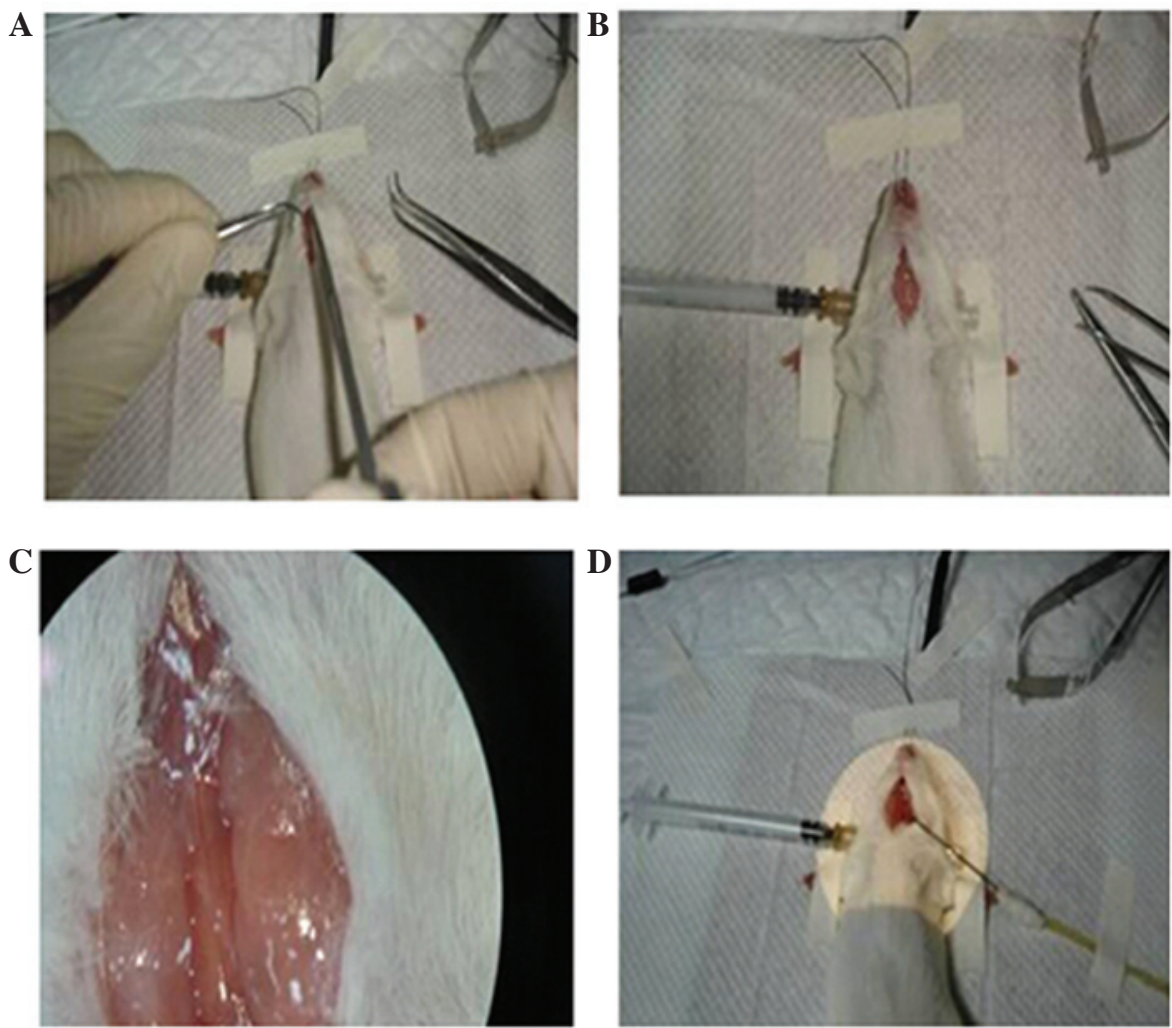

Figure 1. Diagram of the MCAO-induced rat model of stroke. (A) A surgical incision followed the routine disinfection. (B and C) An incision was made into the cranium to expose the MCA. (D) The MCA was occluded by electric coagulation at the end of the main MCA trunk. MCAO, middle cerebral artery occulsion.

than that of the rats in groups $\mathrm{A}$ and $\mathrm{B}$ but with a statistically significant difference $(\mathrm{P}<0.01)$ (Table II).

Correlation of scores. The immobility time of the rats in groups $\mathrm{B}$ and $\mathrm{C}$ in the forced swimming test increased between the 3 rd and 7 th days, while the scores in the other tests decreased; therefore, correlation analysis was performed between the forced swimming test and the other test results of cognitive and motor functions. The results showed that there was a strongly negative correlation between the results of the forced swimming test and the results for the sucrose solution consumption and horizontal and vertical motion tests $(\mathrm{R}=-0.695,-0.758$ and -0.713 , respectively; $\mathrm{P}<0.05)$, which showed that these tests results were highly correlated. The selected tests therefore proved to be suitable indicators for the determination of the behavioral changes in rat models (Table III).

\section{Discussion}

Stroke is a frequently occurring disease among the elderly, although there has been a clear increase in the incidence rate of stroke in young and middle-aged patients (14). The rate of stroke-induced disabilities can reach as high as $80 \%(15)$. Stroke-induced disabilities can have a serious effect on the daily life of the patient, bring great suffering and place a heavy burden on the family and society (16). A number of previous clinical studies have reported that at least $40-50 \%$ of stroke patients are likely to suffer from PSD, lasting between 2 months and 1 year after the stroke. In total, $45.4 \%$ of patients have been found to suffer from PSD in the first month after the stroke, with mild to moderate depression accounting for $91.8 \%$ of all cases of PSD $(17,18)$. The elderly are more susceptible to suffering PSD than young people. An understanding of the prevention and treatment of PSD can improve the self-care ability of stroke patients, reduce the sequelae and complications and help patients integrate back into society; therefore, stroke and its associated complications warrant considerable research.

The causes of post-stroke depression can be biological, psychological or social and are closely associated with genetic factors (19). The pathogenesis may be related to non-specific systemic reactions which are stimulated by various factors, in particular, severe, persistent and unmanageable stimuli can lead to obvious symptoms of disease (20).

There have been several studies on the establishment of stroke and PSD animal models $(5,8)$; however, only a few studies have focused on the behavioral changes in PSD rat models (9). The present study was therefore performed to investigate the behavioral changes in a PSD rat model. We hypothesized that rats with PSD would exhibit reduced activity and interest, despair and other manifestations of depression. Out of the three evaluation indexes in this study, the sucrose solution intake reflected the euphoria associated with food, a natural reward. The reduction of the sucrose solution consumption could not merely be interpreted as a reduction in the animal's sensation of thirst, but was suggestive of anhedonia, one of the core symptoms of human depression. The open-field test was used to detect the directed and exploratory behavior, as well as the state of fear and alertness, of the rats in the new environment. The score of horizontal motion reflected the activity of the animals, while the score of vertical motion reflected the degree of curiosity 
Table I. Test results of the three groups of rats after 3 days.

\begin{tabular}{lccc}
\hline Index & Group A & Group B & Group C \\
\hline Sucrose solution consumption (g) & $28.48 \pm 5.51$ & $25.59 \pm 5.44$ & $19.49 \pm 4.37^{\mathrm{a}, \mathrm{c}}$ \\
Horizontal motion (blocks) & $30.74 \pm 6.59$ & $17.31 \pm 4.32^{\mathrm{a}}$ & $11.98 \pm 3.77^{\mathrm{b}, \mathrm{d}}$ \\
Upright motion (times) & $23.38 \pm 6.11$ & $14.26 \pm 4.81^{\mathrm{a}}$ & $9.27 \pm 2.70^{\mathrm{b}, \mathrm{d}}$ \\
Forced swimming test (sec) & $19.74 \pm 7.15$ & $25.28 \pm 10.47$ & $48.53 \pm 18.17^{\mathrm{b}, \mathrm{d}}$ \\
\hline
\end{tabular}

Values are presented as the mean \pm standard deviation. ${ }^{a} \mathrm{P}<0.05$ and ${ }^{\mathrm{b}} \mathrm{P}<0.01$, vs. group $\mathrm{A}$; ${ }^{\mathrm{c}} \mathrm{P}<0.05$ and ${ }^{\mathrm{d}} \mathrm{P}<0.01$, vs. group $\mathrm{B}$.

Table II. Test results of the three groups of rats after 7 days.

\begin{tabular}{lrrr}
\hline Index & Group A & Group B & Group C \\
\hline Sucrose solution consumption (g) & $29.58 \pm 5.76$ & $20.64 \pm 5.12^{\mathrm{a}}$ & $15.79 \pm 3.78^{\mathrm{b}, \mathrm{c}}$ \\
Horizontal motion (blocks) & $29.53 \pm 6.37$ & $16.57 \pm 4.10^{\mathrm{a}}$ & $9.13 \pm 3.25^{\mathrm{b}, \mathrm{c}}$ \\
Upright motion (times) & $22.87 \pm 6.02$ & $14.58 \pm 4.69^{\mathrm{a}}$ & $7.59 \pm 2.36^{\mathrm{b}, \mathrm{c}}$ \\
Forced swimming test (sec) & $20.31 \pm 7.95$ & $28.77 \pm 13.18^{\mathrm{a}}$ & $57.41 \pm 22.08^{\mathrm{b}, \mathrm{c}}$ \\
\hline
\end{tabular}

Values are presented as the mean \pm standard deviation. ${ }^{\mathrm{a}} \mathrm{P}<0.05$, vs. group $\mathrm{A} ;{ }^{\mathrm{b}} \mathrm{P}<0.05$ and ${ }^{\mathrm{c}} \mathrm{P}<0.01$, vs. group $\mathrm{B}$.

Table III. Analysis of the correlation between the forced swimming test and other indexes.

\begin{tabular}{lccc}
\hline Statistic & Sucrose solution consumption & Horizontal motion & Vertical motion \\
\hline $\mathrm{R}$ & -0.695 & -0.758 & -0.713 \\
P-value & $<0.05$ & $<0.05$ & $<0.05$ \\
\hline
\end{tabular}

of the animals toward novel environmental conditions. The open-field test and sucrose solution consumption indexes are the most common behavioral indexes with which depression is studied, and in this case they were able to prove whether or not our hypothesis was correct. The results indicated that on the 3rd and 7th days, the sucrose solution consumption of the PSD rat model was significantly reduced, suggesting low sensitivity to food reward stimulation, which resembles the anhedonia that occurs in patients with depression. The results of the open-field and forced swimming tests showed that prolonged stimulation caused the activities of the rats in the PSD and stroke groups to decrease more notably. The reduction in the horizontal motion suggested a decrease in the animal activity, while the decrease in the upright motion revealed a reduced curiosity toward the novel environment in the rats of the two groups. These results are evidence that the animals were suffering from depression. The immobility time in the forced swimming test reflected the degree of despair in the rats. The immobility time in the PSD group was far greater than that in the other two groups, suggesting the desperate state exhibited by the rats with PSD. The study results confirmed our hypothesis.

The reduced activity, decreased interest and manifestations of depression in rats with PSD are based on solid physiological biochemical and anatomical evidence. It has previously been indicated that, when a patient suffers from depression, the activity of biogenic amines (serotonin, norepinephrine, dopamine) decreases; by contrast, when a patient suffers from mania, the activity of biogenic amines increases (21). The metabolites of biogenic amines, including 5-hydroxyindoleacetic acid from serotonin, homovanillic acid from dopamine and 3-methoxy-4-hydroxyphenylglycol from norepinephrine, have been shown to vary in the blood, urine and cerebrospinal fluid (22). Recent anatomical studies have shown that damage to the hippocampus is a common pathological change in depression $(23,24)$. Furthermore, animal experiments have shown that, in the rat model of stroke, the survival of new cells in situ in the dentate gyrus and the proportion of cells differentiating into neurons are reduced and the proportion of cells differentiating into glial cells is increased, as compared with the normal rats. It has also been shown that stress can influence the neurogenesis rate in the subgranular zone of the dentate gyrus and that antidepressants can promote neuronal remodeling of the hippocampus to resist depression (25). Furthermore, it has been suggested that anhedonia, decreased interest, psychomotor retardation and other symptoms of depression are associated with the damage to the reward system function in the central nervous system. It has additionally been indicated that the PSD rat model induced by isolation in combination with chronic, unpredictable, mild stress exhibits a series of changes in emotional behavior that, to a certain extent, resembles depression in human. 
The most significant finding of the present study is that, as indicated by the results of the behavioral tests, model rats exhibited certain behavioral characteristics similar to those of anhedonia in human patients, such as reduced interest and psychomotor inhibition. These behavioral changes were sustained, suggesting that the depression model was successful and hence could be used as a model of depression for further research. This successful animal model could be used for drug intervention experiments, to determine the variation in behavior at the level of gene regulation or to observe changes in brain tissue structure or pathophysiological changes in the PSD rat model, so as to lay the foundations for the improvement of the diagnosis and treatment of clinical depression. Such findings would play an important role in the recovery of the cerebral function of stoke patients and enable the evaluation of the curative effect of intervention methods.

The present study did, however, have certain limitations, such as the small number of rat models included into the study and the relatively short observation time (7 days). Studying PSD requires the examination of a larger number of cases and a longer follow-up period, and therefore a precise conclusion cannot be drawn from the present results. In addition, this study was not a completely randomized, prospective, double-blind study; these issues will be addressed in our next study. Despite these limitations, the findings of the present study have served as a reminder that PSD has a serious negative impact on the health of patients and that the treatment of stroke warrants further investigation.

\section{References}

1. Jauch EC, Saver JL, Adams HP Jr, et al; American Heart Association Stroke Council; Council on Cardiovascular Nursing; Council on Peripheral Vascular Disease; Council on Clinical Cardiology: Guidelines for the early management of patients with acute ischemic stroke: A guideline for healthcare professionals from the American Heart Association/American Stroke Association. Stroke 44: 870-947, 2013.

2. Wei YL, Liu ZL and Duan XQ: Analysis of prognostic factors of feeding-swallowing disorder patients in stroke unit. Zhong Guo Kang Fu Yi Xue Za Zhi 25: 322-325, 2010 (In Chinese).

3. Bradberry JC, Fagan SC, Gray DR and Moon YS: New perspectives on the pharmacotherapy of ischemic stroke. J Am Pharm Assoc (2003) 44 (2 Suppl 1): S46-56, 2004.

4. Kim JM, Stewart R, Kang HJ, et al: A prospective study of statin use and poststroke depression. J Clin Psychopharmacol 34: 72-79, 2014

5. Schneider MA and Schneider MD: Recognizing poststroke depression. Nursing 42: 60-63, 2012.

6. Del Zoppo GJ, Saver JL, Jauch EC and Adams HP Jr; American Heart Association Stroke Council: Expansion of the time window for treatment of acute ischemic stroke with intravenous tissue plasminogen activator: A science advisory from the American Heart Association/American Stroke Association. Stroke 40: 2945-2948, 2009.

7. Videbech $\mathrm{P}$ and Ravnkilde B: Hippocampal volume and depression: A meta-analysis of MRI studies. Am J Psychiatry 161: 1957-1966, 2004.
8. Kunze A, Zierath D, Drogomiretskiy O and Becker K: Strain differences in fatigue and depression after experimental stroke. Transl Stroke Res 5: 604-611, 2014

9. Wang SH, Zhang ZJ, Guo YJ, Sui YX and Sun Y: Involvement of serotonin neurotransmission in hippocampal neurogenesis and behavioral responses in a rat model of post-stroke depression. Pharmacol Biochem Behav 95: 129-137, 2010.

10. Tuglu C, Kara SH, Caliyurt O, Vardar E and Abay E: Increased serum tumor necrosis factor-alpha levels and treatment response in major depressive disorder. Psychopharmacology (Berl) 170: 429-433, 2003.

11. Basso AM, Bratcher NA, Harris RR, Jarvis MF, Decker MW and Rueter LE: Behavioral profile of $\mathrm{P} 2 \mathrm{X} 7$ receptor knockout mice in animal models of depression and anxiety: Relevance for neuropsychiatric disorders. Behav Brain Res 198: 83-90, 2008.

12. Spalletta $G$, Bossù $P$, Ciaramella $A$, Bria $P$, Caltagirone $C$ and Robinson RG: The etiology of poststroke depression: A review of the literature and a new hypothesis involving inflammatory cytokines. Mol Psychiatry 11: 984-991, 2006.

13. Craft TK and DeVries AC: Role of IL-1 in poststroke depressive-like behavior in mice. Biol Psychiatry 60: 812-818, 2006.

14. Aarnio K, Haapaniemi E, Melkas S, Kaste M, Tatlisumak T and Putaala J: Long-term mortality after first-ever and recurrent stroke in young adults. Stroke 45: 2670-2676, 2014.

15. Zweckberger K, Juettler E, Bösel J and Unterberg WA: Surgical aspects of decompression craniectomy in malignant stroke: Review. Cerebrovasc Dis 38: 313-323, 2014.

16. Diener HC, Bogousslavsky J, Brass LM, et al; MATCH investigators: Aspirin and clopidogrel compared with clopidogrel alone after recent ischaemic stroke or transient ischaemic attack in high-risk patients (MATCH): Randomised, double-blind, placebo-controlled trial. Lancet 364: 331-337, 2004.

17. Park GY, Im S, Oh CH, Lee SJ and Pae CU: The association between the severity of poststroke depression and clinical outcomes after first-onset stroke in Korean patients. Gen Hosp Psychiatry: Mar 11, 2015 (Epub ahead of print).

18. Henn FA and Vollmayr B: Neurogenesis and depression: Etiology or epiphenomenon? Biol Psychiatry 56: 146-150, 2004.

19. Tharwani HM, Yerramsetty P, Mannlli P, Patkar A and Masand P: Recent advances in poststroke depression. Curr Psychiatry Rep 9: 225-231, 2007.

20. Boggio PS, Amancio EJ, Correa CF, et al: Transcranial DC stimulation coupled with TENS for the treatment of chronic pain: A preliminary study. Clin J Pain 25: 691-695, 2009.

21. Morrissette DA and Stahl SM: Modulating the serotonin system in the treatment of major depressive disorder. CNS Spectr 19 (Suppl 1): 57-67, 2014.

22. Hoffman RE, Gueorguieva R, Hawkins KA, et al: Temporoparietal transcranial magnetic stimulation for auditory hallucinations: Safety, efficacy and moderators in a fifty patient sample. Biol Psychiatry 58: 97-104, 2005.

23. Holmes J: An attachment model of depression: Integrating findings from the mood disorder laboratory. Psychiatry 76: 68-86, 2013.

24. Yang A, Hua P, Shang X, Cui Z, Zhong S, Gong G and William Humphreys G: Deficiency of brain structural sub-network underlying post-ischemic stroke apathy. Eur J Neurol 22: 341-347, 2015.

25. Lefaucheur JP: Stroke recovery can be enhanced by using repetitive transcranial magnetic stimulation (rTMS). Neurophysiol Clin 36: 105-115, 2006. 